\title{
On the Determination of Transition-Moment Directions from Emission Anisotropy Measurements*
}

\author{
A. Kawski and Z. Gryczyński \\ Luminescence Research Group, Institute of Experimental Physics, University of Gdaǹsk, Poland \\ and Institute of Physics, Pedagogical University, Słupsk, Poland
}

Z. Naturforsch. 41 a, 1195-1199 (1986); received June 16, 1986

Based on the theory of photoluminescence polarization, a description of the determination of transition-moment directions in luminescent molecules oriented in a stretched polymer film is given. This method is applied to the linear trans-diphenylpolyenes $\left(\mathrm{C}_{2 \mathrm{~h}}\right.$ symmetry $): 1,8$-diphenyloctatetraene (DPO), 1,6-diphenylhexatriene (DPH) and 1,4-diphenylbutadiene (DPB). The same transition moment polarized along the long axis of DPO, DPH and DPB is responsible for absorption and fluorescence $(\beta \approx 0)$, when exciting in the long wave absorption band. The results are compared with relevant data obtained from dichroism measurements.

\section{Introduction}

In recent years a number of papers report on polarized absorption and luminescence of aromatic molecules aligned in stretched polymer films [1,2]. The orientational mechanism in a streched polymer is more complex than in photoselection. The photoselection technique, in which molecules are selected by exciting a random direction distribution of molecules in dilute vitrified solution by linearly polarized light, yields according to the Perrin equation [3]

$$
r_{0}=\frac{2}{5}\left(\frac{3}{2} \cos ^{2} \beta-\frac{1}{2}\right)
$$

( $\beta$ is the angle between the absorption and emission transition moments); the theoretical limits of the emission anisotropy are $r_{0}=-\frac{1}{5}$ and $r_{0}=\frac{2}{5}$. The photoselection technique is a relative method. The directions of the absorption and emission transition moments themselves are not revealed. A review of investigations in which the principle of the photoselection was employed [4], is given by Dörr [5, 6].

The determination of transition-moment directions requires studies with ordered systems, such as stretched polymer films. In this paper a somewhat different procedure, based on the theory of photoluminescence polarization, is proposed.

Reprint requests to Prof. Dr. A. Kawski, Uniwersytet Gdański, Instytut Fizyki Doświadczalnej, ul. Wita Stwosza 57, 80-952 Gdańsk, Poland.

* This work was carried out under contract CPBP 01.06.

\section{Theory}

Let us consider a prolate molecule with its directions $\boldsymbol{A}$ and $\boldsymbol{E}$ of the absorption and emission oscillators fixed in the molecule (Figure 1). The electric vector $\varepsilon$ of the exciting light may be oriented along the $z$-axis. $I_{\|}$and $I_{\perp}$ designate the components of the fluorescence intensity $\left(I=I_{\|}+2 I_{\perp}\right)$ parallel

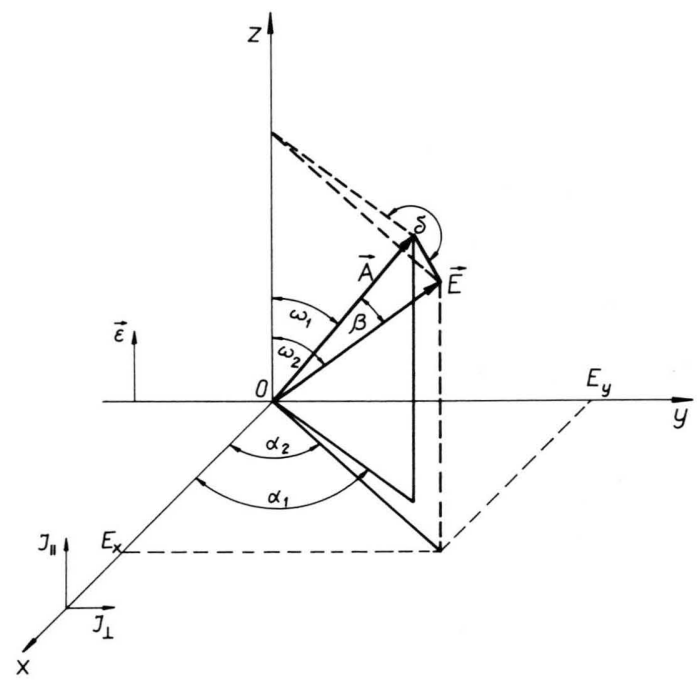

Fig. 1. Geometry of the system. The exciting light $\varepsilon$ is polarized in the $z$ direction. The fluorescence is observed along $x$ (or along $y$ in the case of thin PVA films). The vectors $\boldsymbol{A}$ and $\boldsymbol{E}$ represent the direction of the absorptionand emission-transition moments. $\delta$ is the angle between the planes formed by the $\boldsymbol{z}, \boldsymbol{A}$ and $\boldsymbol{E}, \boldsymbol{A}$ directions. The position of the angles $\omega_{1}, \omega_{2}, \alpha_{1}$ and $\alpha_{2}$ is evident from the figure. 
and perpendicular to $\varepsilon$, respectively, as observed along the $x$-axis. The angles $\omega_{1}, \omega_{2}, \beta$ and $\delta$ (Fig. 1) obey the relation

$$
\cos \omega_{2}=\cos \omega_{1} \cos \beta+\sin \omega_{1} \sin \beta \cos \delta .
$$

On squaring (2) and averaging over the azimuthal angle $\delta\left(\langle\cos \delta\rangle=0,\left\langle\cos ^{2} \delta\right\rangle=\frac{1}{2}\right)$ we obtain to a given absorption oszillator direction $\omega_{1}$ a mean emission oscillator value

$$
\begin{aligned}
\left\langle\cos ^{2} \omega_{2}\right\rangle & =\cos ^{2} \omega_{1} \cos ^{2} \beta+\frac{1}{2} \sin ^{2} \omega_{1} \sin ^{2} \beta \\
& =\left(\frac{3}{2} \cos ^{2} \omega_{1}-\frac{1}{2}\right)\left(\cos ^{2} \beta-\frac{1}{3}\right)+\frac{2}{6},
\end{aligned}
$$

where $\beta$ is constant for a given transition in the molecule.
Hence, the distribution of molecules with rod shape excited by vertically polarized light $\varepsilon$ (Fig. 1) is given by

$$
f\left(\omega_{1}\right) \mathrm{d} \omega_{1}=f_{\mathrm{g}}\left(\omega_{1}\right) \cos ^{2} \omega_{1} \mathrm{~d} \omega_{1} .
$$

For $R_{\mathrm{s}}=1$, the probability distribution (8) determines the maximum selection which can be obtained using optical excitation of an isotropic solution. The emission anisotropy in a vitrified dilute solution is a product of the loss of anisotropy due to photoselection $(2 / 5)$ and that due to the angular displacement of the absorption, $\boldsymbol{A}$, and emission, $\boldsymbol{E}$, dipoles. Hence, from (5) we obtain (1).

For $R_{\mathrm{s}}>1$, from (5), (6) and (8) we obtain

$$
r\left(\beta, R_{\mathrm{s}}\right)=\left\{\frac{3}{2} \frac{\left(a^{2}-1\right)^{1 / 2}+2 a^{2}\left(a^{2}-1\right)^{-1 / 2}-3 a^{2} \arcsin \frac{1}{a}}{2\left(a^{2}-1\right)^{-1 / 2}-2 \arcsin \frac{1}{a}}-\frac{1}{2}\right\}\left(\frac{3}{2} \cos ^{2} \beta-\frac{1}{2}\right),
$$

Averaging over all directions $\omega_{1}$ gives the mean $\left\langle\cos ^{2} \omega_{2}\right\rangle$ which is connected with the emission anisotropy $r\left(\omega_{2}\right)[7]$ :

$$
r\left(\omega_{2}\right)=\frac{3}{2} \frac{I_{1}}{I}-\frac{1}{2}=\frac{3}{2}\left\langle\cos ^{2} \omega_{2}\right\rangle-\frac{1}{2} .
$$

Substitution of the averaged equation (3) into (4) yields

$$
r\left(\beta, \omega_{1}\right)=\left(\frac{3}{2}\left\langle\cos ^{2} \omega_{1}\right\rangle-\frac{1}{2}\right)\left(\frac{3}{2} \cos ^{2} \beta-\frac{1}{2}\right),
$$

where

$$
\left\langle\cos ^{2} \omega_{1}\right\rangle=\frac{\int_{0}^{\pi / 2} \cos ^{2} \omega_{1} \cdot f\left(\omega_{1}\right) \mathrm{d} \omega_{1}}{\int_{0}^{\pi / 2} f\left(\omega_{1}\right) \mathrm{d} \omega_{1}}
$$

and $f\left(\omega_{1}\right) \mathrm{d} \omega_{1}$ is the direction distribution in the excited state owing to the polarized absorption.

For molecules being in the ground state, Tanizaki [8] derived the following distribution function of the oriented rods with respect to a stretching direction (z-axis):

$f_{\mathrm{g}}\left(\omega_{1}\right)=R_{\mathrm{s}}^{2} \sin \omega_{1}\left\{1+\left(R_{\mathrm{s}}^{2}-1\right) \sin ^{2} \omega_{1}\right\}^{-3 / 2}$,

where $R_{\mathrm{s}}$ is the stretch ratio*.

* The stretch ratio $R_{\mathrm{s}}$ is defined as the axial ratio $a / b$ ( $a$ and $b$ are the semi-major and -minor axes) of an ellipse deformed from a circle of radius $r$ which was initially drawn on the film. Uniform stretching of the film was tested at each $R_{\mathrm{s}}$ by comparing an imaginary volume of $4 \pi a \cdot b^{2} / 3$ with the initial volume of $4 \pi r^{3} / 3$. These two volumes agreed within an experimental uncertainty of $4 \%$ [9]. where

$$
a=\frac{R_{\mathrm{s}}^{2}}{R_{\mathrm{s}}^{2}-1} .
$$

Plotting $r\left(\beta, R_{\mathrm{s}}\right)$ against $R_{\mathrm{s}}$ for various values of $\beta(0 \leqq \beta \leqq \pi / 2)$ a family of curves representing (9) are obtained as shown in Figure 2.

\section{Experimental}

In order to test the validity of (9), the following linear trans-diphenyl-polyenes $\left(\mathrm{C}_{2 \mathrm{~h}}\right.$ symmetry $) \mathrm{Ph}$ $(\mathrm{CH}=\mathrm{CH})_{n}-\mathrm{Ph}$ were chosen: 1,8-diphenyloctatetraene (DPO) $(n=4), \quad 1,6$-diphenylhexatriene (DPH) $(n=3)$ and 1,4-diphenylbutadiene (DPB) $(n=2)$. Their structural formulas and directions of the transition moments are shown in Figure 3.

It is known that the fluorescence transition in $\mathrm{DPO}$ and DPH occurs from the low-lying ${ }^{1} \mathrm{~A}_{\mathrm{g}}^{*}$ state $\left(\mathrm{S}_{1}\right)$ and in DPB from the ${ }^{1} \mathrm{~B}_{\mathrm{u}}^{*}$ state $\left(\mathrm{S}_{2}\right)$, which is at slightly higher energy [10].

For these molecules imbedded in stretched polymer films the direction of the electronic transition moments has been determined by the linear dichroism and then by the method based on (9).

\subsection{Samples and methods}

Isotropic films were made of a $15 \%$ aqueous solution of polyvinyl alcohol (PVA) in which the lumi- 


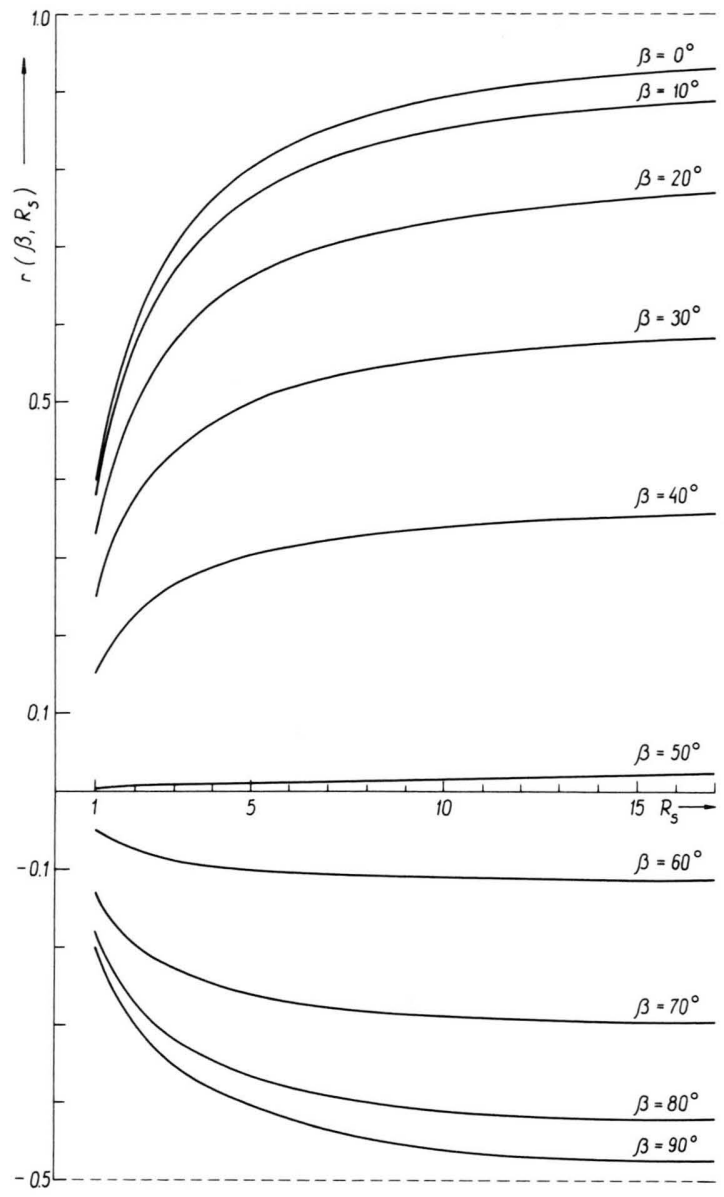

Fig. 2. Theoretical dependence of $r\left(\beta, R_{\mathrm{s}}\right)$ on the stretch $R_{\mathrm{s}}$ for various values of $\beta$.

$$
\text { CH }
$$

Fig. 3. Structural formulas and the directions (double pointed arrows) of transition moments of trans-diphenylpolyenes.

nescent molecules DPO DPH and DPB were set up by methanol. The method of obtaining the films was described early [11-13]. PVA films were stretched at about $350 \mathrm{~K}$, the rate of stretching being controlled. The quality of obtained film samples was found to be essentially affected by temperature and the stretching rate. For the measurements the most homogeneous films were selected.

The polarized absorption measurements were carried out using a single-beam VSU-2P spectrophotometer. The use of a parallel light beam and a Glan prism enabled a high degree of polarization to be achieved $(P>0.98)$. The fluorescence anisotropy measurements were carried out by means of a home-built measuring apparatus [14].

\subsection{Dichroism of chromophores in stretched PVA films}

In order to determine the transitions directions of the molecular electronic bands in the stretched PVA films from the dichroic absorption spectra, Tanizaki $[8,12]$ proposed the following expression for the density ratio $R_{\mathrm{d}}=D_{\|} / D_{\perp}$ (where $D_{\|}$and $D_{\perp}$ are optical densities for completely and linearly polarized lights whose electric vectors are respectively parallel and perpendicular to the stretching direction) as a function of the stretch ratio $R_{\mathrm{s}}$ :

$$
R_{\mathrm{d}}=\frac{2+2\left(2 m^{2}-1\right) T}{2 m^{2}+1-\left(2 m^{2}-1\right) T}
$$

where

$$
\begin{aligned}
& T=\frac{R_{\mathrm{s}}^{2}}{R_{\mathrm{s}}^{2}-1} \\
& \cdot\left\{1-\left[\frac{\pi}{2}-\arctan \left(R_{\mathrm{s}}^{2}-1\right)^{-1 / 2}\right]\left(R_{\mathrm{s}}^{2}-1\right)^{-1 / 2}\right\}
\end{aligned}
$$

and

$$
\alpha=\operatorname{arccot} m
$$

is the angle between the direction of the transition moment and the orientation axis.

The measured absorption spectra of DPO, DPH and DPB for the $D_{\|}$and $D_{\perp}$ components in the PVA film stretched 5-fold $\left(R_{\mathrm{s}}=5^{3 / 2}\right)$ are shown in Figure 4 . In the long-wave absorption bands for DPO $\left(22000-30000 \mathrm{~cm}^{-1}\right)$, DPH $\left(24000-32000 \mathrm{~cm}^{-1}\right)$ and DPB $\left(26000-34000 \mathrm{~cm}^{-1}\right) R_{\mathrm{d}}$ remains constant. The values of $m$ for DPO, DPH and DPB were obtained from a comparison of the measured $R_{\mathrm{d}}$ versus $R_{\mathrm{s}}$ values with the theoretical curves calculated according to (10) (see Figure 5). $m$ amounts to 13,11 and 8 in the investigated bands of DPO, DPH and DPB, thus yielding the angles $\alpha=4.4^{\circ}$, $5,2^{\circ}$ and $7.2^{\circ}$, respectively. It follows that the first long-wave absorption bands for the investigated 


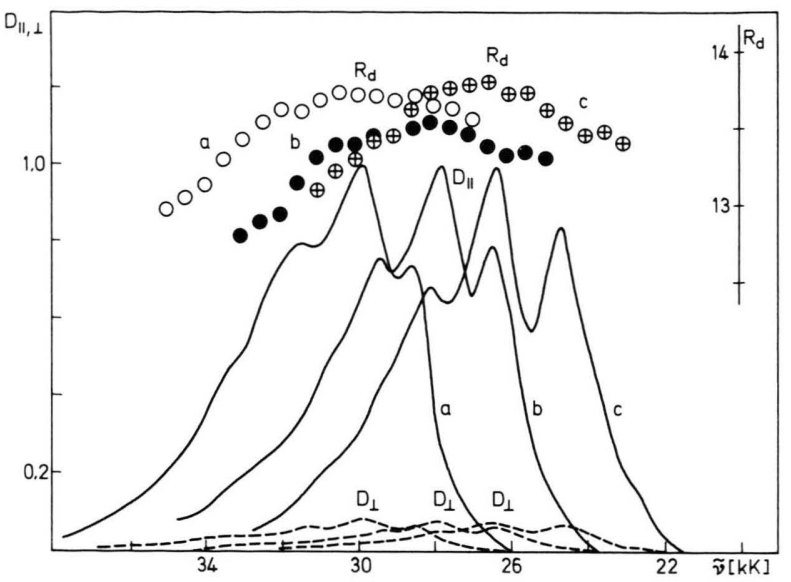

Fig. 4. Absorption spectra of a) DPB, b) DPH, and c) DPO for the $D$ and $D \perp$ components in PVA film stretched 5-fold, and the dependence of $R_{\mathrm{d}}=D_{\|} / D_{\perp}$ vs. wavenumber $\boldsymbol{v}$.

molecules exhibits polarization along the long axis of these molecules, as shown in Figure 3.

\subsection{Fluorescence anisotropy of chromophores in stretched PVA films}

The measured limiting fluorescence anisotropies $r_{0}$ for DPO, DPH and DPB in isotropic PVA films are $0.354,0.360$ and 0.330 , respectively. The difference between the fundamental fluorescence anisotropy $\left(r_{\mathrm{f}}=0.4\right)$ and the limiting value $\left(r_{0}\right)$ is due to the depolarization of torsional vibrations which occur immediately following excitation during the thermal relaxation of the luminescent molecule $[15,16]$.

The measured values of $r$ for DPO, DPH and DPB in stretched PVA films are shown in Figure 6. The values lie between the theoretical curves calculated according to (9) for the angles $\beta 0^{\circ}$ and $10^{\circ}$. For DPO, DPH and DPB the angles $\beta$ are $6^{\circ}$, $8^{\circ}$ and $11^{\circ}$, respectively. For molecules having a large long-axis-short-axis ratio $\left(\mathrm{C}_{2 \mathrm{~h}}\right.$ symmetry) the obtained angles $\beta$ are small, because the molecules rigidly embedded in cavities between the closely spaced polymer chains are considerably oriented in a stretched polymer film. The DPB molecule is not well oriented in the stretching direction of the PVA sheet. Nevertheless, it is possible to draw conclusions about the angle $\beta$.

Our results show that the transition from the excited singlet state to the ground state is polarized along the long axis of these molecules and the same

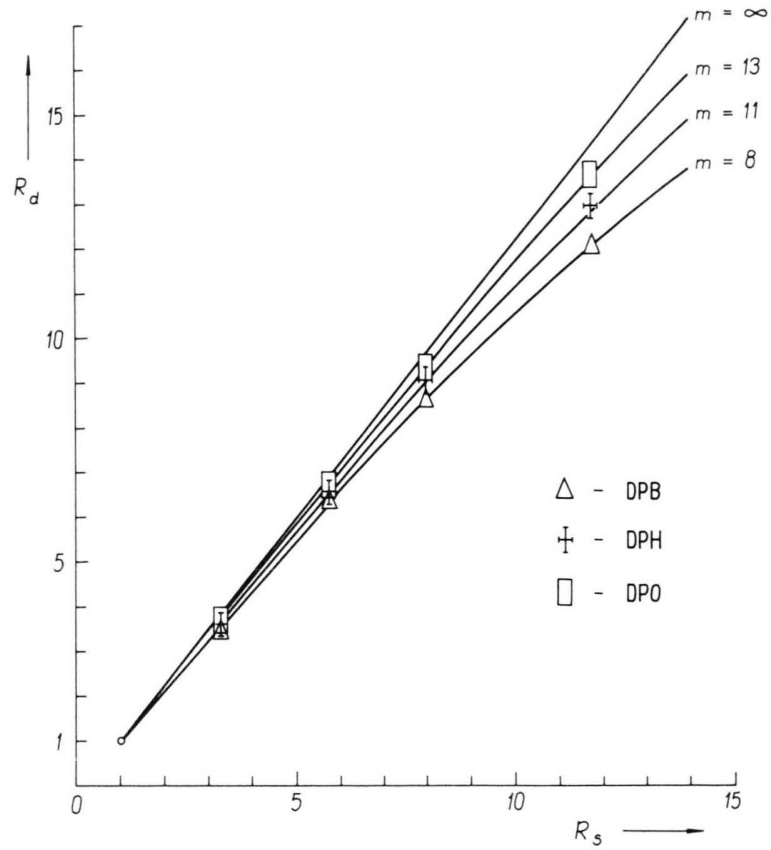

Fig. 5. Theoretical curves of $R_{\mathrm{d}}$ vs. the stretch ratio $R_{\mathrm{s}}$ for different values of $m$ and the corresponding measured $m$ values $(\square-\mathrm{DPO}, \bigcirc-\mathrm{DPH}, \triangle-\mathrm{DPB})$.

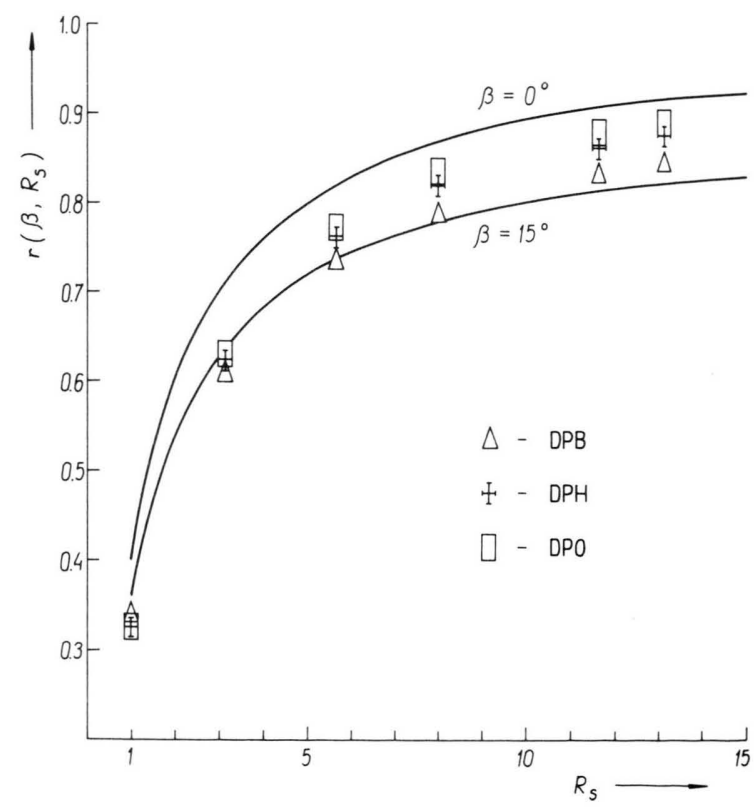

Fig. 6. Dependence of $r\left(\beta, R_{\mathrm{s}}\right)$ on the degree of stretching $R_{\mathrm{S}}$ for DPO (O), DPH (+) and DPB ( $\left.\square\right)$. The full lines are the theoretical curves according to (9) for $\beta=0^{\circ}$ and $\beta=$ $15^{\circ}$. The measured $r$ values were determined by the excitation wavenumbers $26178 \mathrm{~cm}^{-1}, 27933 \mathrm{~cm}^{-1}$ and $29990 \mathrm{~cm}^{-1}$ for DPO, DPH and DPB, respectively. 
transition moment is responsible for absorption and for emission $(\beta \approx 0)$, when exciting in the long wave absorption band. Polarization of transitions to high-

[1] B. Norden, App. Spectr. Rev. 14, 157 (1978) and references cited therein.

[2] J. J. Dekkers, Emission Spectroscopy of Aromatic Hydrocarbons in Stretched Polymer Films and in Shpolskii Martices, Vrije Universiteit te Amsterdam, 1979 and references sited therein.

[3] F. Perrin, Ann. Physique 12, 169 (1929)

[4] A. C. Albrecht, J. Molec. Spectr. 6, 84 (1961).

[5] F. Dörr, Angew. Chemie 5, 478 (1966).

[6] F. Dörr, in: "Creation and Detection of the Excited State", ed. A. A. Lamola, Vol. 1, Dekker, New York 1971, Chapter 2.

[7] A. Kawski, J. Kamiński, and J. Kukielski, Z. Naturforsch. 34 a, 702 (1979)

[8] Y. Tanizaki, Bull. Chem. Soc. Japan 38, 1798 (1965). er excited states $\left(\mathrm{S}_{n}\right)$ can be determined with this method by varying the wavelength of the exciting light.

[9] Y. Matsuoka, K. Yamaoka, Bull. Chem. Soc. Japan 52, 3163 (1979).

[10] D. J. S. Birch and R. E. Imhof, Chem. Phys. Letters, 88, 243 (1982).

[11] J. J. Dekkers, G. Ph. Hoornweg, C. MacLean, and N. H. Velthorst, Chem. Phys. Letters, 19, 517 (1973).

[12] Y. Tanizaki, Bull. Chem. Soc. Japan 32, 75 (1959)

[13] I. Gryczyński, Z. Gryczyński, A. Kawski, and S. Paszyc, Photochem. Photobiol. 39, 319 (1984).

[14] A. Kawski, Z. Kojro, and A. Kubicki, Z. Naturforsch. 40 a, 313 (1985).

[15] A. Jabłoński, Acta Phys. Polon. 10,33, 193 (1950).

[16] A. Kawski, A. Kubicki, I. Weyna, and I. Janić, Z. Naturforsch. 40 a, 559 (1985). 